\title{
FINITELY PRESENTED AND COHERENT ORDERED MODULES AND RINGS
}

\author{
F. WEHRUNG
}

\begin{abstract}
We extend the usual definition of coherence, for modules over rings, to partially ordered right modules over a large class of partially ordered rings, called po-rings. In this situation, coherence is equivalent to saying that solution sets of finite systems of inequalities are finitely generated semimodules. Coherence for ordered rings and modules, which we call po-coherence, has the following features:

(i) Every subring of $\mathbb{Q}$, and every totally ordered division ring, is po-coherent.

(ii) For a partially ordered right module $\boldsymbol{A}$ over a po-coherent poring $\boldsymbol{R}, \boldsymbol{A}$ is po-coherent if and only if $\boldsymbol{A}$ is a finitely presented $\boldsymbol{R}$-module and $\boldsymbol{A}^{+}$is a finitely generated $\boldsymbol{R}^{+}$-semimodule.

(iii) Every finitely $p o$-presented partially ordered right module over a right po-coherent po-ring is po-coherent.

(iv) Every finitely presented abelian lattice-ordered group is pocoherent.
\end{abstract}

\section{INTRODUCTION}

Let $\boldsymbol{A}$ be a partially ordered right module over a partially ordered $\operatorname{ring} \boldsymbol{R}$. We say that $\boldsymbol{A}$ is po-coherent, if the solution sets of finite systems of inequalities over $\boldsymbol{A}$ with unknowns in $\boldsymbol{R}$ are finitely generated semimodules over $\boldsymbol{R}^{+}$, that is, monoids closed under multiplication by scalars in $\boldsymbol{R}^{+}$. This extends the classical definition of coherence for modules.

In order to avoid pathologies and unwieldy statements, we restrict our attention to the quite general class of partially ordered right modules over po-rings, see Definition 1.1. In particular, every totally ordered ring is a po-ring. It turns out that for those modules, the classical notion of finitely presented structure (see Definition 3.1), that we shall denote here by finitely po-presented to emphasize the presence of the partial ordering, can conveniently be replaced by the slightly

1991 Mathematics Subject Classification. 06F25, 16W80, 12J15, 15A39, 08C15.

Key words and phrases. Ring, module, ordered, finitely presented, finitely related, coherent, system of inequalities, matrix. 
stronger notion of finitely related structure (see Definition 3.2). Then we say that a partially ordered right module $\boldsymbol{A}$ over a $p o$-ring $\boldsymbol{R}$ is po-coherent (see Definition 3.2), if every finitely generated submodule (endowed with the induced ordering) is finitely related. Equivalently, solution sets of finite homogeneous systems of inequalities are finitely generated semimodules (see Theorem [3.6).

In the case where $\boldsymbol{R}$ is, itself, right po-coherent (that is, po-coherent as a partially ordered right module over itself), the characterization of finitely $p o$-presented structures takes a much more wieldy form:

Theorem 4.2. $\boldsymbol{A}$ is finitely po-presented if and only if $\boldsymbol{A}$ is finitely related, if and only if $\boldsymbol{A}$ is finitely presented as a $\boldsymbol{R}$-module and $\boldsymbol{A}^{+}$is finitely generated as a $\boldsymbol{R}^{+}$-semimodule.

Another particular feature of partially ordered modules over a pocoherent po-ring is the following:

Theorem 4.1. Let $\boldsymbol{R}$ be a po-ring, let $\boldsymbol{A}$ be a partially ordered right $\boldsymbol{R}$-module. If $\boldsymbol{R}$ is right po-coherent and $\boldsymbol{A}$ is finitely po-presented, then $\boldsymbol{A}$ is po-coherent.

The supply of right po-coherent po-rings is quite large:

Theorem 6.4. Every subring of $\mathbb{Q}$ is po-coherent.

The main difficulty encountered in the proof of this result consists of providing a proof for the ring of integers. However, this is, basically, well-known, and it follows for example from much more general results due to Grillet (see [6]) and Effros, Handelman, and Shen (see 4]) about certain partially ordered abelian groups called dimension groups. A similar result can be proved for "dimension vector spaces" over a totally ordered field (or division ring). A consequence of the proof of this result is that every totally ordered division ring is a po-coherent poring (Corollary [7.3).

The supply of po-coherent modules is also quite large:

Theorem 8.1. Every finitely presented abelian lattice-ordered group, viewed as a partially ordered abelian group, is po-coherent.

Our proof of this result uses the classical representation of finitely presented abelian lattice-ordered groups as groups of piecewise linear functions on polyhedral cones, see 7] for a survey.

The proofs of the results of this paper are, basically, easy, especially when they are expressed with the matrix formalism introduced in Section 2. However, the results that they prove are, apparently, nontrivial, 
and they provide, moreover, a convenient common platform for handling a number of different, but related, results that would, otherwise, require separate proofs. Examples of such statements can be found in 3]. Furthermore, as mentioned above, the classical notion of a finitely presented structure is not the most convenient in our context of ordered structures, which seems to require this switch to finitely related structures. By Theorem 4.2, the difference between finitely po-presented and finitely related vanishes anyway for ordered modules over $p o$-coherent rings, which are the cases that matter for us.

\section{BASIC CONCEPTS}

The objects that we shall consider in this paper are, basically, rings (always associative and unital), and (always unital) right modules over those rings. Rings and modules will be denoted by capitalized boldface roman characters, such as $\boldsymbol{R}, \boldsymbol{S}, \boldsymbol{A}, \boldsymbol{B}$, while matrices will be denoted by capitalized lightface roman characters, such as $M, N, P, Q, X$. Elements of a given module will usually be denoted by lightface lower case roman characters, such as $a, b, x$, while scalars in a given ring will be denoted by lightface lower case greek letters, such as $\alpha, \beta, \xi$. Since we shall consider right modules, matrices and module homomorphisms will be written on the left.

We shall put $\mathbb{N}=\mathbb{Z}^{+} \backslash\{0\}$. If $\boldsymbol{A}$ is a right module over a ring $\boldsymbol{R}$ and if $\boldsymbol{S}$ is a subset of $\boldsymbol{A}$, then we shall denote by $\mathfrak{M}_{m, n}(\boldsymbol{S})$ the set of all matrices with entries in $\boldsymbol{S}$, with $m$ rows and $n$ columns, for all $m$, $n \in \mathbb{Z}^{+}$. Furthermore, we shall put $\mathfrak{M}_{n}(\boldsymbol{S})=\mathfrak{M}_{n, n}(\boldsymbol{S})$. Thus, for all $p, q, r \in \mathbb{Z}^{+}$, the product operation of matrices takes any pair $\langle X, Y\rangle$, where $X \in \mathfrak{M}_{p, q}(\boldsymbol{A})$ and $Y \in \mathfrak{M}_{q, r}(\boldsymbol{R})$, to a matrix $X Y \in \mathfrak{M}_{p, r}(\boldsymbol{A})$. If $a$ is an element of $\boldsymbol{A}$, then we shall often identify the $1 \times 1$ matrix $(a)$ with the element $a$ itself.

Our rings and modules will be partially ordered. The topic of the paper is, in fact, designed to cover the context of totally ordered rings and partially ordered right modules over these rings. All the partially ordered rings we shall work with will be thus defined as follows:

Definition 1.1. A po-ring is a ring $\langle\boldsymbol{R},+, \cdot, 0,1\rangle$, endowed with a partial ordering $\leq$ such that the following conditions hold:

(i) $\langle\boldsymbol{R},+, \cdot, 0,1, \leq\rangle$ is a partially ordered ring, that is, $x \leq y$ implies that $x+z \leq y+z$ and, if $z \geq 0, x z \leq y z$ and $z x \leq z y$, for all $x, y, z \in \boldsymbol{R}$.

(ii) $\boldsymbol{R}$ is directed, that is, $\boldsymbol{R}=\boldsymbol{R}^{+}+\left(-\boldsymbol{R}^{+}\right)$.

(iii) $\boldsymbol{R}$ satisfies $0 \leq 1$. 
Note, in particular, that every totally ordered ring is a po-ring.

We shall consider partially ordered right modules over po-rings. For example, every partially ordered $\operatorname{ring} \boldsymbol{R}$ is also a partially ordered right module over itself (and a partially ordered left module as well).

One can define in a classical fashion quotients of partially ordered right modules by convex submodules, see, for example, [9, page 122].

If $\boldsymbol{B}$ is a submodule of $\boldsymbol{A}$, then we shall put

$$
\text { Conv } \boldsymbol{B}=\{z \in \boldsymbol{A} \mid \exists x, y \in \boldsymbol{B}, x \leq z \leq y\} .
$$

It is easy to see that Conv $\boldsymbol{B}$ is the convex submodule of $\boldsymbol{A}$ generated by $\boldsymbol{B}$. A finitely generated convex submodule of $\boldsymbol{A}$ is a submodule of the form Conv $\boldsymbol{B}$, where $\boldsymbol{B}$ is a finitely generated submodule of $\boldsymbol{A}$.

Our next section, Section 2, will be devoted to set a convenient computational framework, based on matrices, for handling systems of equations and inequalities over partially ordered right modules. This is done, mainly, in order to avoid carrying everywhere large amounts of indices which would make the notations considerably heavier.

\section{Matricial REPRESEntations of Systems of EQUATIONS AND INEQUALITIES}

We shall fix in this section a po-ring $\boldsymbol{R}$ and a partially ordered right $\boldsymbol{R}$-module $\boldsymbol{A}$. In the whole paper, we shall basically consider homogeneous systems of equations and inequalities. The typical form of a system of inequalities is the following:

$$
\left\{\begin{aligned}
a_{11} \xi_{1}+\cdots+a_{1 n} \xi_{n} & \geq 0 \\
a_{21} \xi_{1}+\cdots+a_{2 n} \xi_{n} & \geq 0 \\
\vdots \quad & \vdots \\
a_{m 1} \xi_{1}+\cdots+a_{m n} \xi_{n} & \geq 0
\end{aligned}\right.
$$

where the elements $a_{i j}$ belong to $\boldsymbol{A}$ and the elements $\xi_{j}$ belong to $\boldsymbol{R}$. Note that the element $a_{i 1} \xi_{1}+\cdots+a_{i n} \xi_{n}$ belongs to $\boldsymbol{A}$, for all $i \in\{1, \ldots, m\}$.

Now we define matrices $M \in \mathfrak{M}_{m, n}(\boldsymbol{A})$ and $X \in \mathfrak{M}_{n, 1}(\boldsymbol{R})$ as follows:

$$
M=\left(\begin{array}{cccc}
a_{11} & a_{12} & \ldots & a_{1 n} \\
a_{21} & a_{22} & \ldots & a_{2 n} \\
\vdots & \vdots & \ddots & \vdots \\
a_{m 1} & a_{m 2} & \ldots & a_{m n}
\end{array}\right), \quad \text { and } \quad X=\left(\begin{array}{c}
\xi_{1} \\
\xi_{2} \\
\vdots \\
\xi_{n}
\end{array}\right)
$$

Then the system (2.1) can be written under the following simple form:

$$
M X \geq 0
$$


If we think of $M$ as given, and $X$ as representing the matrix of unknowns of the system (2.1), then it is natural that we wish to define the notion of a general solution of (2.1), and thus of (2.2). Denote by $\mathcal{S}$ the solution set of (2.2), that is,

$$
\mathcal{S}=\left\{X \in \mathfrak{M}_{n, 1}(\boldsymbol{R}) \mid M X \geq 0\right\} .
$$

Then $\mathcal{S}$ is a $\boldsymbol{R}^{+}$-subsemimodule of $\mathfrak{M}_{n, 1}(\boldsymbol{R})$, that is, an additive submonoid closed under scalar multiplication by elements of $\boldsymbol{R}^{+}$. Similar considerations hold for systems of equations. The relevant information may be recorded as follows:

Lemma 2.1. Let $n \in \mathbb{N}$, let $\mathcal{S}$ be a subset of $\mathfrak{M}_{n, 1}(\boldsymbol{R})$. Then $\mathcal{S}$ is a finitely generated right $\boldsymbol{R}$-submodule (resp., right $\boldsymbol{R}^{+}$-subsemimodule) of $\mathfrak{M}_{n, 1}(\boldsymbol{R})$ if and only if there exist $k \in \mathbb{N}$ and a matrix $S \in \mathfrak{M}_{n, k}(\boldsymbol{R})$ such that

$$
\begin{aligned}
\mathcal{S} & =\left\{S Y \mid Y \in \mathfrak{M}_{k, 1}(\boldsymbol{R})\right\} \\
(\text { resp., } \mathcal{S} & \left.=\left\{S Y \mid Y \in \mathfrak{M}_{k, 1}\left(\boldsymbol{R}^{+}\right)\right\}\right)
\end{aligned}
$$

We shall also consider in this paper systems similar to

$$
\left\{\begin{array}{r}
M X \geq 0 \\
X \geq 0
\end{array}\right.
$$

which may be viewed as analogues of (2.2) in the case where $M$ has entries in $\boldsymbol{R} \cup \boldsymbol{A}$. We shall call such systems mixed systems.

\section{Finitely RELATED AND po-COHERENT PARTially ORDERED MODULES}

If $\boldsymbol{R}$ is a po-ring and if $\boldsymbol{A}$ is a partially ordered right $\boldsymbol{R}$-module, we say that a matrix $M$ with entries in $\boldsymbol{A}$ is spanning, if the entries of $M$ generate $\boldsymbol{A}$ as a $\boldsymbol{R}$-submodule. Of particular importance in this work will be the class of spanning row matrices, where a row matrix is, by definition, a matrix with only one row.

Let us first define, in our context, finitely po-presented structures:

Definition 3.1. Let $\boldsymbol{R}$ be a po-ring, let $\boldsymbol{A}$ be a partially ordered right $\boldsymbol{R}$-module.

(i) Let $n \in \mathbb{N}$, let $U \in \mathfrak{M}_{1, n}(\boldsymbol{A})$. We say that $\boldsymbol{A}$ is finitely popresented at $U$, if the solution set in $\mathfrak{M}_{n, 1}(\boldsymbol{R})$ of the system $U X \geq 0$ is a finitely generated right $\boldsymbol{R}^{+}$-subsemimodule of $\mathfrak{M}_{n, 1}(\boldsymbol{R})$. 
(ii) $\boldsymbol{A}$ is finitely po-presented, if $\boldsymbol{A}$ is finitely po-presented at some spanning row matrix $U$ of elements of $\boldsymbol{A}$ (in particular, $\boldsymbol{A}$ is a finitely generated right $\boldsymbol{R}$-module).

In our context of po-rings, this definition agrees with the classical definition of finitely presented structures in terms of generators and relations; see, below, the remark (i) following Definition 3.2.

More relevant to us will be the following stronger definition of a finitely related structure:

Definition 3.2. Let $\boldsymbol{R}$ be a po-ring, let $\boldsymbol{A}$ be a partially ordered right $\boldsymbol{R}$-module.

(i) Let $n \in \mathbb{N}$, let $U \in \mathfrak{M}_{1, n}(\boldsymbol{A})$. We say that $\boldsymbol{A}$ is finitely related at $U$, if the solution set in $\mathfrak{M}_{n, 1}(\boldsymbol{R})$ of the mixed system

$$
\left\{\begin{aligned}
U X & \geq 0 \\
X & \geq 0
\end{aligned}\right.
$$

is a finitely generated right $\boldsymbol{R}^{+}$-subsemimodule of $\mathfrak{M}_{n, 1}(\boldsymbol{R})$.

(ii) $\boldsymbol{A}$ is finitely related, if $\boldsymbol{A}$ is a finitely generated right $\boldsymbol{R}$-module, and $\boldsymbol{A}$ is finitely related at every spanning row matrix $U$ of elements of $\boldsymbol{A}$.

(iii) $\boldsymbol{A}$ is po-coherent, if every finitely generated submodule of $\boldsymbol{A}$ is finitely related; that is, if $\boldsymbol{A}$ is finitely related at every row matrix of elements of $\boldsymbol{A}$.

Say that $\boldsymbol{R}$ is a right po-coherent po-ring if $\boldsymbol{R}$, viewed as a partially ordered right $\boldsymbol{R}$-module, is po-coherent.

Of course, one can define similarly po-coherent left modules over a po-ring, and left po-coherent po-rings. A po-ring is po-coherent, if it is both right and left po-coherent.

Several remarks are in order.

(i) Definition [3.1] agrees with the classical definition given in universal algebra, stated, for example, in [8] (page 32 for the general definition of an algebraic system, and page 223 for the definition of a finitely presented algebraic system). The verification of this easy exercise strongly uses the fact that $\boldsymbol{R}$ is directed. For example, this is already used at the simplest level, to verify that the solution set of the inequality $(0) \cdot(x) \geq 0$ should be a finitely generated right subsemimodule (and not just submodule) of $\boldsymbol{R}$. This holds, in particular, if $\boldsymbol{R}$ is directed.

(ii) The terminology coherent is also inspired from classical module theory: 
Definition 3.3. Let $\boldsymbol{R}$ be a ring.

(a) Let $\boldsymbol{A}$ be a right $\boldsymbol{R}$-module. Say that $\boldsymbol{A}$ is coherent, if every finitely generated submodule of $\boldsymbol{A}$ is finitely presented.

(b) Say that $\boldsymbol{R}$ is a right coherent ring, if $\boldsymbol{R}$, viewed as a right $\boldsymbol{R}$-module, is coherent.

This terminology differs slightly from the one used in 10, where it is supposed, in addition, that $\boldsymbol{A}$ is itself finitely presented. However, for rings, both terminologies are equivalent.

(iii) It may seem slightly awkward to introduce the different but very similar definitions of finitely po-presented and finitely related partially ordered right $\boldsymbol{R}$-module. However, we shall see that in all the cases that will matter to us, these definitions are equivalent (see Theorem 4.2).

The general connection between finitely po-presented and finitely related partially ordered right $\boldsymbol{R}$-modules is the following:

Proposition 3.4. Let $\boldsymbol{R}$ be a po-ring. Then every finitely related partially ordered right $\boldsymbol{R}$-module is finitely po-presented.

Proof. Let $\boldsymbol{A}$ be a finitely related partially ordered right $\boldsymbol{R}$-module. Let $n \in \mathbb{N}$, let $U \in \mathfrak{M}_{1, n}(\boldsymbol{A})$ be a spanning row matrix of $\boldsymbol{A}$. Since $\boldsymbol{A}$ is finitely related and the matrix $(U-U)$ is a spanning row matrix of $\boldsymbol{A}$, there exist $k \in \mathbb{N}$ and $P, Q \in \mathfrak{M}_{n, k}\left(\boldsymbol{R}^{+}\right)$such that the matrices $Y$, $Z \in \mathfrak{M}_{n, 1}\left(\boldsymbol{R}^{+}\right)$which satisfy $U Y-U Z \geq 0$ are exactly the matrices of the form $Y=P T, Z=Q T$ for some $T \in \mathfrak{M}_{k, 1}\left(\boldsymbol{R}^{+}\right)$. Now, consider an element $X \in \mathfrak{M}_{n, 1}(\boldsymbol{R})$. Since $\boldsymbol{R}$ is directed, there are $Y, Z \in \mathfrak{M}_{n, 1}\left(\boldsymbol{R}^{+}\right)$ such that $X=Y-Z$; it follows that $U X \geq 0$ if and only if $U Y-U Z \geq$ 0 . Thus, the matrices $X \in \mathfrak{M}_{n, 1}(\boldsymbol{R})$ such that $U X \geq 0$ are exactly the matrices of the form $(P-Q) T$, where $T \in \mathfrak{M}_{k, 1}\left(\boldsymbol{R}^{+}\right)$.

Remark. For all the cases that will matter to us, the converse of Proposition 3.4 will hold; see Theorem 4.2

By using the formalism of Section 2, we can easily recover, in a self-contained fashion, the well-known result that finite presentability as defined above does not depend on the generating subset, see V.11, Corollary 7, p. 223 in [8]:

Proposition 3.5. Let $\boldsymbol{A}$ be a finitely generated partially ordered right $\boldsymbol{R}$-module. Then $\boldsymbol{A}$ is finitely po-presented if and only if $\boldsymbol{A}$ is finitely po-presented at every spanning row matrix of $\boldsymbol{A}$.

Proof. We prove the nontrivial direction. So let $U$ and $V$ be spanning row matrices of $\boldsymbol{A}$, with, say, $U \in \mathfrak{M}_{1, m}(\boldsymbol{A})$ and $V \in \mathfrak{M}_{1, n}(\boldsymbol{A})$. Since 
each of the matrices $U$ and $V$ has its entries lying in the $\boldsymbol{R}$-submodule generated by the entries of the other, there are matrices $M \in \mathfrak{M}_{m, n}(\boldsymbol{R})$ and $N \in \mathfrak{M}_{n, m}(\boldsymbol{R})$ such that $V=U M$ and $U=V N$. Suppose that $\boldsymbol{A}$ is finitely po-presented at $U$, that is, there are $k \in \mathbb{N}$ and $S \in \mathfrak{M}_{m, k}(\boldsymbol{R})$ such that

$$
\left\{X \in \mathfrak{M}_{m, 1}(\boldsymbol{R}) \mid U X \geq 0\right\}=\left\{S Z \mid Z \in \mathfrak{M}_{k, 1}\left(\boldsymbol{R}^{+}\right)\right\}
$$

Now let $Y \in \mathfrak{M}_{n, 1}(\boldsymbol{R})$ such that $V Y \geq 0$. This can be written $U M Y \geq$ 0, thus, by (3.1), there exists $Z \in \mathfrak{M}_{k, 1}\left(\boldsymbol{R}^{+}\right)$such that $M Y=S Z$. Thus $N M Y=N S Z$. Furthermore, since $\boldsymbol{R}$ is directed, there are $Y_{0}$, $Y_{1} \in \mathfrak{M}_{n, 1}\left(\boldsymbol{R}^{+}\right)$such that $Y=Y_{0}-Y_{1}$. Therefore, the equality

$$
\begin{aligned}
Y & =(Y-N M Y)+N M Y \\
& =\left(I_{n}-N M\right) Y_{0}+\left(N M-I_{n}\right) Y_{1}+N S Z
\end{aligned}
$$

holds, where $I_{n}$ denotes the identity matrix of order $n$. Conversely, the equalities $V\left(I_{n}-N M\right)=V-V N M=0$ and $V N S=U S \geq 0$ hold, thus every matrix $Y$ of the form (3.2) satisfies $V Y \geq 0$. It follows that

$$
\begin{aligned}
& \left\{Y \in \mathfrak{M}_{n, 1}(\boldsymbol{R}) \mid V Y \geq 0\right\}= \\
& \left\{\left(I_{n}-N M\right) Y_{0}+\left(N M-I_{n}\right) Y_{1}+N S Z \mid\right. \\
& \left.Y_{0}, Y_{1} \in \mathfrak{M}_{n, 1}\left(\boldsymbol{R}^{+}\right) \text {and } Z \in \mathfrak{M}_{k, 1}\left(\boldsymbol{R}^{+}\right)\right\},
\end{aligned}
$$

so that $\boldsymbol{A}$ is also finitely po-presented at $V$.

The following result gives a characterization of coherence for partially ordered modules:

Theorem 3.6. Let $\boldsymbol{R}$ be a po-ring, let $\boldsymbol{A}$ be a partially ordered right $\boldsymbol{R}$-module. Then $\boldsymbol{A}$ is po-coherent if and only if for all $k, m, n \in \mathbb{Z}^{+}$ and all $M \in \mathfrak{M}_{k, m}(\boldsymbol{A}), N \in \mathfrak{M}_{k, n}(\boldsymbol{A})$, the solution set of the following mixed system

$$
\left\{\begin{aligned}
M X+N Y & \geq 0 \\
X & \geq 0,
\end{aligned}\right.
$$

with unknowns $X \in \mathfrak{M}_{m, 1}(\boldsymbol{R})$ and $Y \in \mathfrak{M}_{n, 1}(\boldsymbol{R})$, is a finitely generated right $\boldsymbol{R}^{+}$-semimodule.

Proof. We prove the nontrivial direction. So, suppose that $\boldsymbol{A}$ is pocoherent. We shall first prove a claim:

Claim. For all $m, n \in \mathbb{N}$ and all $M \in \mathfrak{M}_{m, n}(\boldsymbol{A})$, the solution set in $\mathfrak{M}_{n, 1}(\boldsymbol{R})$ of the mixed system $\{M X \geq 0 ; \quad X \geq 0 \quad$ is a finitely generated right $\boldsymbol{R}^{+}$-subsemimodule of $\mathfrak{M}_{n, 1}(\boldsymbol{R})$. 
Proof of Claim. We argue by induction on $m$. For $m=1$, the conclusion is just the assumption on $\boldsymbol{A}$. Suppose the claim is true for $m$. Let $M \in \mathfrak{M}_{m+1, n}(\boldsymbol{A})$, and consider the mixed system

$$
\left\{\begin{array}{r}
M X \geq 0 \\
X \geq 0 .
\end{array}\right.
$$

Decompose $M$ into blocks, as $M=\left(\begin{array}{l}N \\ U\end{array}\right)$, where $N \in \mathfrak{M}_{m, n}(\boldsymbol{A})$ and $U \in \mathfrak{M}_{1, n}(\boldsymbol{A})$. In particular, the mixed system (3.4) is equivalent to the following mixed system:

$$
\left\{\begin{aligned}
N X & \geq 0 \\
U X & \geq 0 \\
X & \geq 0 .
\end{aligned}\right.
$$

By assumption, the solution set of the mixed system consisting of the last two subsystems of (3.5) above is a finitely generated right $\boldsymbol{R}^{+}$subsemimodule of $\mathfrak{M}_{n, 1}(\boldsymbol{R})$, that is, there exist $p \in \mathbb{N}$ and $S \in \mathfrak{M}_{n, p}(\boldsymbol{R})$ such that

$$
\left\{X \in \mathfrak{M}_{n, 1}\left(\boldsymbol{R}^{+}\right) \mid U X \geq 0\right\}=\left\{S Y \mid Y \in \mathfrak{M}_{p, 1}\left(\boldsymbol{R}^{+}\right)\right\} .
$$

Note, in particular, that $S \geq 0$, and that $N S$ belongs to $\mathfrak{M}_{m, p}(\boldsymbol{A})$. By induction hypothesis, there are $q \in \mathbb{N}$ and $P \in \mathfrak{M}_{p, q}(\boldsymbol{R})$ such that

$$
\left\{Y \in \mathfrak{M}_{p, 1}\left(\boldsymbol{R}^{+}\right) \mid N S Y \geq 0\right\}=\left\{P Z \mid Z \in \mathfrak{M}_{q, 1}\left(\boldsymbol{R}^{+}\right)\right\} .
$$

Note, in particular, that $P \geq 0$. It follows then easily from (3.6) and (3.7) that the solution set of the mixed system (3.5) in $\mathfrak{M}_{n, 1}(\boldsymbol{R})$ is equal to

$$
\left\{S P Z \mid Z \in \mathfrak{M}_{q, 1}\left(\boldsymbol{R}^{+}\right)\right\},
$$

which establishes the induction step.

Claim.

Now we can conclude the proof of Theorem 3.6. We consider the mixed system

$$
\left\{\begin{array}{r}
M X+N Y_{0}-N Y_{1} \geq 0 \\
\left(\begin{array}{c}
X \\
Y_{0} \\
Y_{1}
\end{array}\right) \geq 0,
\end{array}\right.
$$

with unknowns $X \in \mathfrak{M}_{m, 1}(\boldsymbol{R})$ and $Y_{0}, Y_{1} \in \mathfrak{M}_{n, 1}(\boldsymbol{R})$. By the Claim, there are $l \in \mathbb{N}$ and matrices $P \in \mathfrak{M}_{m, l}\left(\boldsymbol{R}^{+}\right), Q_{0}, Q_{1} \in \mathfrak{M}_{n, l}\left(\boldsymbol{R}^{+}\right)$such 
that the solution set of (3.8) consists of all matrices of the form

$$
\left(\begin{array}{c}
P T \\
Q_{0} T \\
Q_{1} T
\end{array}\right),
$$

for $T \in \mathfrak{M}_{l, 1}\left(\boldsymbol{R}^{+}\right)$. But every $Y \in \mathfrak{M}_{n, 1}(\boldsymbol{R})$ can be written as a difference $Y=Y_{0}-Y_{1}$ where $Y_{0}, Y_{1} \in \mathfrak{M}_{n, 1}\left(\boldsymbol{R}^{+}\right)$. Hence the solution set of (3.3) consists of all matrices of the form

$$
\left(\begin{array}{c}
P T \\
\left(Q_{0}-Q_{1}\right) T
\end{array}\right)
$$

for $T \in \mathfrak{M}_{l, 1}\left(\boldsymbol{R}^{+}\right)$.

Corollary 3.7. Let $\boldsymbol{R}$ be a po-ring, let $\boldsymbol{A}$ be a partially ordered right $\boldsymbol{R}$-module. If $\boldsymbol{A}$ is po-coherent, then $\boldsymbol{A}$ is coherent.

Proof. Let $n \in \mathbb{N}$, let $U \in \mathfrak{M}_{1, n}(\boldsymbol{A})$. We must prove that the set

$$
\mathcal{S}=\left\{X \in \mathfrak{M}_{n, 1}(\boldsymbol{R}) \mid U X=0\right\}
$$

is a finitely generated right $\boldsymbol{R}$-module. Since the system $U X=0$ is equivalent to the system

$$
\left(\begin{array}{c}
U \\
-U
\end{array}\right) X \geq 0
$$

this follows immediately from Theorem 3.6.

\section{RIGHT po-COHERENT po-RINGS}

This section is devoted to the study of a few characterizations of pocoherent partially ordered right modules. We start with the following result.

Theorem 4.1. Let $\boldsymbol{R}$ be a po-ring, let $\boldsymbol{A}$ be a partially ordered right $\boldsymbol{R}$-module. If $\boldsymbol{R}$ is right po-coherent and $\boldsymbol{A}$ is finitely po-presented, then $\boldsymbol{A}$ is po-coherent.

Proof. We first establish two claims:

Claim 1. $\boldsymbol{A}$ is finitely related.

Proof of Claim. By assumption, $\boldsymbol{A}$ is finitely generated. Let $U$ be a spanning row matrix of $\boldsymbol{A}$, say, $U \in \mathfrak{M}_{1, n}(\boldsymbol{A})$, where $n \in \mathbb{N}$. By Proposition 3.5. $\boldsymbol{A}$ is finitely $p o$-presented at $U$, that is, there are $p \in \mathbb{N}$ and $S \in \mathfrak{M}_{n, p}(\boldsymbol{R})$ such that

$$
\left\{X \in \mathfrak{M}_{n, 1}(\boldsymbol{R}) \mid U X \geq 0\right\}=\left\{S Y \mid Y \in \mathfrak{M}_{p, 1}\left(\boldsymbol{R}^{+}\right)\right\} .
$$


Note, in particular, that $S$ is a matrix with entries in $\boldsymbol{R}$. By Theorem [3.6. since $\boldsymbol{R}$ is right po-coherent, there exist $q \in \mathbb{N}$ and $T \in$ $\mathfrak{M}_{p, q}\left(\boldsymbol{R}^{+}\right)$such that

$$
\left\{Y \in \mathfrak{M}_{p, 1}\left(\boldsymbol{R}^{+}\right) \mid S Y \geq 0\right\}=\left\{T Z \mid Z \in \mathfrak{M}_{q, 1}\left(\boldsymbol{R}^{+}\right)\right\} .
$$

Note, in particular, that $T \geq 0$ and $S T \geq 0$. It follows easily from (4.1) and (4.2) that the equality

$$
\left\{X \in \mathfrak{M}_{n, 1}\left(\boldsymbol{R}^{+}\right) \mid U X \geq 0\right\}=\left\{S T Z \mid Z \in \mathfrak{M}_{q, 1}\left(\boldsymbol{R}^{+}\right)\right\}
$$

holds. Therefore, $\boldsymbol{A}$ is finitely related at $U$.

Claim 1.

Claim 2. Every finitely generated submodule of $\boldsymbol{A}$ is finitely po-presented.

Proof of Claim. Let $\boldsymbol{B}$ be a finitely generated submodule of $\boldsymbol{A}$. Let $U$ (resp., $V$ ) be a spanning row matrix of $\boldsymbol{A}$ (resp., $\boldsymbol{B}$ ), say, $U \in \mathfrak{M}_{1, m}(\boldsymbol{A})$ and $V \in \mathfrak{M}_{1, n}(\boldsymbol{B})$. Since $\boldsymbol{B}$ is contained in $\boldsymbol{A}$, there exists a matrix $M \in \mathfrak{M}_{m, n}(\boldsymbol{R})$ such that $V=U M$. We define sets $\mathcal{S}$ and $\mathcal{T}$ of matrices as follows:

$$
\mathcal{S}=\left\{X \in \mathfrak{M}_{m, 1}(\boldsymbol{R}) \mid U X \geq 0\right\}, \quad \mathcal{T}=\left\{Y \in \mathfrak{M}_{n, 1}(\boldsymbol{R}) \mid V Y \geq 0\right\} .
$$

By assumption on $\boldsymbol{A}, \mathcal{S}$ is a finitely generated $\boldsymbol{R}^{+}$-subsemimodule of $\mathfrak{M}_{m, 1}(\boldsymbol{R})$, that is, there exist $k \in \mathbb{N}$ and $S \in \mathfrak{M}_{m, k}(\boldsymbol{R})$ such that $\mathcal{S}=\left\{S Z \mid Z \in \mathfrak{M}_{k, 1}\left(\boldsymbol{R}^{+}\right)\right\}$. Now consider the following mixed system:

$$
\left\{\begin{aligned}
M Y-S Z & =0 \\
Z & \geq 0
\end{aligned}\right.
$$

with unknowns $Y$ in $\mathfrak{M}_{n, 1}(\boldsymbol{R})$ and $Z$ in $\mathfrak{M}_{k, 1}(\boldsymbol{R})$. Since $\boldsymbol{R}$ is right pocoherent, there are, by Theorem 3.6. $l \in \mathbb{N}$ and matrices $P \in \mathfrak{M}_{n, l}(\boldsymbol{R})$, $Q \in \mathfrak{M}_{k, l}(\boldsymbol{R})$ such that the solution set of (4.3) equals the set

$$
\left\{\left(\begin{array}{l}
P \\
Q
\end{array}\right) T \mid T \in \mathfrak{M}_{l, 1}\left(\boldsymbol{R}^{+}\right)\right\}
$$

But a matrix $Y \in \mathfrak{M}_{n, 1}(\boldsymbol{R})$ belongs to $\mathcal{T}$ if and only if $V Y \geq 0$, that is, $U M Y \geq 0$, which can be written $M Y \in \mathcal{S}$, or: there is a matrix $Z$ such that $Y$ and $Z$ satisfy (4.3). It follows that $\mathcal{T}=$ $\left\{P T \mid T \in \mathfrak{M}_{l, 1}\left(\boldsymbol{R}^{+}\right)\right\}$. This proves that $\mathcal{T}$ is a finitely generated $\boldsymbol{R}^{+}$-subsemimodule of $\mathfrak{M}_{n, 1}(\boldsymbol{R})$.

Claim 2.

We can now conclude the proof of Theorem 4.1. Let $\boldsymbol{B}$ be a finitely generated submodule of $\boldsymbol{A}$. By Claim 2, $\boldsymbol{B}$ is finitely po-presented. By Claim 1, applied to $\boldsymbol{B}, \boldsymbol{B}$ is finitely related. 
We can now give a characterization of finite $p o$-presentability that separates the roles of the algebraic structure and of the ordering:

Theorem 4.2. Let $\boldsymbol{R}$ be a right po-coherent po-ring. For every partially ordered right $\boldsymbol{R}$-module $\boldsymbol{A}$, the following are equivalent:

(i) $\boldsymbol{A}$ is finitely po-presented.

(ii) $\boldsymbol{A}$ is finitely related.

(iii) $\boldsymbol{A}$ is a finitely presented right $\boldsymbol{R}$-module, and $\boldsymbol{A}^{+}$is a finitely generated right $\boldsymbol{R}^{+}$-semimodule.

Proof. (i) $\Rightarrow$ (ii) follows immediately from Theorem 4.1 .

(ii) $\Rightarrow$ (i) has been proved in Proposition 3.4

(i) $\Rightarrow$ (iii) Suppose that (i) holds. By Theorem 4.1, $\boldsymbol{A}$ is po-coherent. By Corollary [3.7, $\boldsymbol{A}$ is coherent. Since $\boldsymbol{A}$ is finitely po-presented, it is finitely generated. It follows that $\boldsymbol{A}$ is finitely presented.

It remains to prove that $\boldsymbol{A}^{+}$is finitely generated over $\boldsymbol{R}^{+}$. By assumption, there are $n \in \mathbb{N}$ and a spanning row matrix $U \in \mathfrak{M}_{1, n}(\boldsymbol{A})$ at which $\boldsymbol{A}$ is finitely po-presented. Hence, there exist $k \in \mathbb{N}$ and $S \in \mathfrak{M}_{n, k}(\boldsymbol{R})$ such that

$$
\left\{X \in \mathfrak{M}_{n, 1}(\boldsymbol{R}) \mid U X \geq 0\right\}=\left\{S Y \mid Y \in \mathfrak{M}_{k, 1}\left(\boldsymbol{R}^{+}\right)\right\} .
$$

Since $U$ is a spanning row matrix of $\boldsymbol{A}$, every element of $\boldsymbol{A}$ has the form $U X$ where $X \in \mathfrak{M}_{n, 1}(\boldsymbol{R})$. Hence, by (4.4), every element of $\boldsymbol{A}^{+}$has the form $U S Y$, where $Y \in \mathfrak{M}_{k, 1}\left(\boldsymbol{R}^{+}\right)$; thus it is a positive linear combination of the finite set $T=\left\{U S E_{l} \mid 1 \leq l \leq k\right\}$, where $\left\langle E_{l} \mid 1 \leq l \leq k\right\rangle$ denotes the canonical basis of $\mathfrak{M}_{k, 1}(\boldsymbol{R})$. Since $0 \leq 1$ in $\boldsymbol{R}$, the elements $U S E_{l}$, for $1 \leq l \leq k$, all belong to $\boldsymbol{A}^{+}$; thus $T$ is a finite generating subset of $\boldsymbol{A}^{+}$.

(iii) $\Rightarrow$ (i) Suppose that (iii) holds. Let $m, n \in \mathbb{N}$ such that there exists a finite generating subset $\left\{a_{1}, \ldots, a_{m}\right\}$ of $\boldsymbol{A}$ as a right $\boldsymbol{R}$-module, and a finite generating subset $\left\{b_{1}, \ldots, b_{n}\right\}$ of $\boldsymbol{A}^{+}$as a right $\boldsymbol{R}^{+}$-semimodule. We define row matrices $U$ and $V$ by

$$
U=\left(\begin{array}{lll}
a_{1} & \cdots & a_{m}
\end{array}\right) \quad \text { and } \quad V=\left(\begin{array}{lll}
b_{1} & \cdots & b_{n}
\end{array}\right) .
$$

We can assume that $\boldsymbol{A}$ is finitely presented at $U$. Since the entries of $U$ generate $\boldsymbol{A}$ as a right $\boldsymbol{R}$-module, there exists $M \in \mathfrak{M}_{m, n}(\boldsymbol{R})$ such that $V=U M$. Since $\boldsymbol{A}$ is finitely presented at $U$, the solution set $\mathcal{S}$ in $\mathfrak{M}_{m, 1}(\boldsymbol{R})$ of the matrix equation $U X=0$ is a finitely generated right $\boldsymbol{R}$-submodule of $\mathfrak{M}_{m, 1}(\boldsymbol{R})$, thus, since $\boldsymbol{R}$ is directed, it is also a finitely generated right $\boldsymbol{R}^{+}$-subsemimodule of $\mathfrak{M}_{m, 1}(\boldsymbol{R})$.

Put $\mathcal{P}=\left\{X \in \mathfrak{M}_{m, 1}(\boldsymbol{R}) \mid U X \geq 0\right\}$. To conclude the proof, it suffices to prove that $\mathcal{P}$ is a finitely generated right $\boldsymbol{R}^{+}$-subsemimodule 
of $\mathfrak{M}_{m, 1}(\boldsymbol{R})$. Since $\mathcal{S}$ is a finitely generated right $\boldsymbol{R}^{+}$-subsemimodule of $\mathfrak{M}_{m, 1}(\boldsymbol{R})$, it suffices to establish the following formula:

$$
\mathcal{P}=\left\{M Y \mid Y \in \mathfrak{M}_{n, 1}\left(\boldsymbol{R}^{+}\right)\right\}+\mathcal{S} .
$$

The containment from the right into the left follows from the fact that $U M=V \geq 0$. Conversely, let $X \in \mathfrak{M}_{m, 1}(\boldsymbol{R})$ such that $U X \geq 0$. Since the entries of $V$ generate $\boldsymbol{A}^{+}$as a right $\boldsymbol{R}^{+}$-subsemimodule, there exists $Y \in \mathfrak{M}_{n, 1}\left(\boldsymbol{R}^{+}\right)$such that $U X=V Y$. This can be written $U X=U M Y$, so that $X-M Y \in \mathcal{S}$. This completes the proof of (4.5).

Remark. Unlike (i) $\Rightarrow$ (ii), several implications in the proof of Theorem 4.2 do not use the full strength of the hypothesis that $\boldsymbol{R}$ is a right $p o$-coherent $p o$-ring. For example, (ii) $\Rightarrow(\mathrm{i})$ and (iii) $\Rightarrow(\mathrm{i})$ hold for arbitrary po-rings.

\section{Coherence Preservation Properties For ORDERED MODULES AND RINGS}

Lemma 5.1. Let $\boldsymbol{R}$ be a po-ring, let $\boldsymbol{A}$ be a partially ordered right $\boldsymbol{R}$-module. If $\boldsymbol{A}$ is po-coherent, then the set of all finitely generated $\boldsymbol{R}$-modules (resp., $\boldsymbol{R}^{+}$-subsemimodules) of $\boldsymbol{A}$ is closed under finite intersection.

Remark. The part about $\boldsymbol{R}$-modules is not really a property of partially ordered modules, and it holds, in fact, in the case where $\boldsymbol{A}$ is a coherent module over a ring $\boldsymbol{R}$.

Proof. We give a proof for the semimodule part; the proof for modules is similar. Let $\boldsymbol{B}$ and $\boldsymbol{C}$ be finitely generated $\boldsymbol{R}^{+}$-subsemimodules of $\boldsymbol{A}$. Let $U$ (resp., $V$ ) be a spanning row matrix of $\boldsymbol{B}$ (resp., $\boldsymbol{C}$ ), say, $U \in \mathfrak{M}_{1, m}(\boldsymbol{B})$ and $V \in \mathfrak{M}_{1, n}(\boldsymbol{C})$. Since $\boldsymbol{A}$ is po-coherent, the solution set of the following mixed system

$$
\left\{\begin{array}{r}
U X-V Y=0 \\
X \geq 0 \\
Y \geq 0
\end{array}\right.
$$

with unknowns $X \in \mathfrak{M}_{m, 1}(\boldsymbol{R})$ and $Y \in \mathfrak{M}_{n, 1}(\boldsymbol{R})$, is, by Theorem 3.6. a finitely generated $\boldsymbol{R}^{+}$-subsemimodule of $\mathfrak{M}_{m, 1}(\boldsymbol{R}) \times \mathfrak{M}_{n, 1}(\boldsymbol{R})$, that is, there exist $k \in \mathbb{N}$ and matrices $P \in \mathfrak{M}_{m, k}\left(\boldsymbol{R}^{+}\right), Q \in \mathfrak{M}_{n, k}\left(\boldsymbol{R}^{+}\right)$such that

$$
\left\{\left(\begin{array}{l}
X \\
Y
\end{array}\right) \in \mathfrak{M}_{m+n, 1}\left(\boldsymbol{R}^{+}\right) \mid U X=V Y\right\}=\left\{\left(\begin{array}{c}
P Z \\
Q Z
\end{array}\right) \mid Z \in \mathfrak{M}_{k, 1}\left(\boldsymbol{R}^{+}\right)\right\} .
$$


In particular, $U P=V Q$. If we denote this matrix by $S$, it is then clear that

$$
\boldsymbol{B} \cap \boldsymbol{C}=\left\{S Z \mid Z \in \mathfrak{M}_{k, 1}\left(\boldsymbol{R}^{+}\right)\right\} .
$$

Lemma 5.2. Let $\boldsymbol{R}$ be a right po-coherent po-ring. Then $\boldsymbol{R}^{n}$, partially ordered coordinatewise, is a po-coherent partially ordered right $\boldsymbol{R}$-module, for all $n \in \mathbb{Z}^{+}$.

Proof. This follows immediately from the definition of po-coherence, and Theorem 3.6 .

We deduce the following preservation property:

Proposition 5.3. Let $\boldsymbol{R}$ be a right po-coherent po-ring. Then every direct sum of po-coherent partially ordered right $\boldsymbol{R}$-modules, partially ordered coordinatewise, is po-coherent.

Proof. Let $\left\langle\boldsymbol{A}_{i} \mid i \in I\right\rangle$ be a family of po-coherent partially ordered right $\boldsymbol{R}$-modules; put $\boldsymbol{A}=\bigoplus_{i \in I} \boldsymbol{A}_{i}$. Let $n \in \mathbb{N}$, let $a_{1}, \ldots, a_{n}$ be elements of $\boldsymbol{A}$. Let $J$ be the union of the supports of all the $a_{k}$; so, $J$ is a finite subset of $I$. We write $a_{k}=\left\langle a_{i, k} \mid i \in I\right\rangle$, for all $k \in\{1, \ldots, n\}$; in particular, $a_{i, k}=0$ if $i \in I \backslash J$. It follows that for all $\xi_{1}, \ldots, \xi_{n} \in \boldsymbol{R}^{+}$, $\sum_{k=1}^{n} a_{k} \xi_{k} \geq 0$ if and only if $\sum_{k=1}^{n} a_{i, k} \xi_{k} \geq 0$, for all $i \in J$. Since $\boldsymbol{A}_{i}$ is po-coherent, the set of all $\left\langle\xi_{1}, \ldots, \xi_{n}\right\rangle \in\left(\boldsymbol{R}^{+}\right)^{n}$ satisfying the inequality $\sum_{k=1}^{n} a_{i, k} \xi_{k} \geq 0$ is a finitely generated $\boldsymbol{R}^{+}$-subsemimodule of $\left(\boldsymbol{R}^{+}\right)^{n}$. We conclude the proof by Lemmas 5.1 and 5.2 .

Corollary 5.4. Let $\boldsymbol{R}$ be a right po-coherent po-ring. Then every free right $\boldsymbol{R}$-module, endowed with the componentwise ordering, is a po-coherent partially ordered right $\boldsymbol{R}$-module.

It is obvious that every submodule of a po-coherent partially ordered module is $p o$-coherent. The situation is slightly more complicated for quotients by convex submodules. In particular, we prove in Example 8.6 that the quotient of a po-coherent partially ordered abelian group by a convex, directed subgroup may not be $p o$-coherent.

We shall now see that this problem does not arise for quotients by finitely generated convex submodules.

Proposition 5.5. Let $\boldsymbol{R}$ be a po-ring, let $\boldsymbol{A}$ be a partially ordered right $\boldsymbol{R}$-module, let $\boldsymbol{F}$ be a finitely generated convex submodule of $\boldsymbol{A}$. If $\boldsymbol{A}$ is po-coherent, then $\boldsymbol{A} / \boldsymbol{F}$ is po-coherent.

Proof. Let $\boldsymbol{B}$ be a finitely generated submodule of $\boldsymbol{A}$ such that $\boldsymbol{F}=$ Conv $\boldsymbol{B}$ (see Section 11). Furthermore, denote by $\pi$ the natural projection from $\boldsymbol{A}$ onto $\boldsymbol{A} / \boldsymbol{F}$. Let $V$ be a spanning row matrix of $\boldsymbol{B}$, say, $V \in \mathfrak{M}_{1, n}(\boldsymbol{B})$, for some $n \in \mathbb{N}$. 
Let $\bar{U}$ be a row matrix in $\boldsymbol{A} / \boldsymbol{F}$, say, $\bar{U} \in \mathfrak{M}_{1, m}(\boldsymbol{A} / \boldsymbol{F})$, for some $m \in$ $\mathbb{N}$. There exists $U \in \mathfrak{M}_{1, m}(\boldsymbol{A})$ such that $\bar{U}=\pi U$. We must prove that the solution set in $\mathfrak{M}_{m, 1}(\boldsymbol{R})$ of the mixed system $\{\bar{U} X \geq 0 ; \quad X \geq 0$, is a finitely generated $\boldsymbol{R}^{+}$-subsemimodule of $\mathfrak{M}_{m, 1}(\boldsymbol{R})$.

Consider the following mixed system, with unknowns $X \in \mathfrak{M}_{m, 1}(\boldsymbol{R})$ and $Y \in \mathfrak{M}_{n, 1}(\boldsymbol{R})$ :

$$
\left\{\begin{aligned}
U X+V Y & \geq 0 \\
X & \geq 0 .
\end{aligned}\right.
$$

Since $\boldsymbol{A}$ is po-coherent, there are, by Theorem 3.6. matrices $P \in$ $\mathfrak{M}_{m, k}(\boldsymbol{R})$ and $Q \in \mathfrak{M}_{n, k}(\boldsymbol{R})$ such that the solutions of (5.1) are exactly the matrices of the form $\left(\begin{array}{c}P Z \\ Q Z\end{array}\right)$, where $Z \in \mathfrak{M}_{k, 1}\left(\boldsymbol{R}^{+}\right)$. But for all $X \in \mathfrak{M}_{m, 1}\left(\boldsymbol{R}^{+}\right), \bar{U} X \geq 0$ if and only if there exists $b \in \boldsymbol{F}$ such that $U X+b \geq 0$, that is, there exists $Y$ such that $X$ and $Y$ satisfy (5.1). Therefore, the elements $X$ of $\mathfrak{M}_{m, 1}\left(\boldsymbol{R}^{+}\right)$such that $\bar{U} X \geq 0$ are exactly the matrices of the form $P Z$, where $Z \in \mathfrak{M}_{k, 1}\left(\boldsymbol{R}^{+}\right)$.

We now turn to preservation results for rings:

Proposition 5.6. Let $\boldsymbol{R}$ be a right po-coherent po-ring. Then the matrix ring $\mathfrak{M}_{m}(\boldsymbol{R})$, ordered componentwise, is right po-coherent, for all $m \in \mathbb{N}$.

Proof. Put $\boldsymbol{S}=\mathfrak{M}_{m}(\boldsymbol{R})$. Let $n \in \mathbb{N}$, let $a_{1}, \ldots, a_{n} \in \boldsymbol{S}$. For all $\xi_{1}, \ldots$, $\xi_{n} \in \boldsymbol{S}^{+}$, the condition $\sum_{j=1}^{n} a_{j} \xi_{j} \geq 0$ can be expressed by a system of inequalities, with coefficients all the $n m^{2}$ entries of all the $a_{j}$, and unknowns all the $n m^{2}$ entries of all the $\xi_{j}$. Since $\boldsymbol{R}$ is right $p o$-coherent, the solution set of this system is, by Theorem 3.6, a finitely generated $\boldsymbol{R}^{+}$-subsemimodule of $\mathfrak{M}_{n, 1}(\boldsymbol{S})$. Since it is also a $\boldsymbol{S}^{+}$-subsemimodule of $\mathfrak{M}_{n, 1}(\boldsymbol{S})$, it is, a fortiori, a finitely generated $\boldsymbol{S}^{+}$-subsemimodule of $\mathfrak{M}_{n, 1}(\boldsymbol{S})$.

We shall make use in Section [6 of the following result.

Proposition 5.7. Let $\boldsymbol{S}$ be a po-ring, let $\boldsymbol{R}$ be a directed subring of $\boldsymbol{S}$. We define subsets $\boldsymbol{D}$ and $\boldsymbol{U}$ of $\boldsymbol{R}$ as follows:

$$
\begin{aligned}
& \boldsymbol{D}=\left\{d \in \boldsymbol{R}^{+} \mid \forall x \in \boldsymbol{S}, d x \geq 0 \Rightarrow x \geq 0\right\}, \\
& \boldsymbol{U}=\left\{u \in \boldsymbol{R}^{+} \mid \exists v \in \boldsymbol{S}^{+}, u v=1\right\} .
\end{aligned}
$$

We suppose that for all $s \in \boldsymbol{S}$, there exist $d \in \boldsymbol{D}$ and $u \in \boldsymbol{U}$ such that $d s \in \boldsymbol{R}$ and $s u \in \boldsymbol{R}$.

Then the following holds: if $\boldsymbol{R}$ is right po-coherent, then $\boldsymbol{S}$ is right po-coherent. 
Proof. We start with a claim:

Claim. Let $n \in \mathbb{N}$, let $X \in \mathfrak{M}_{1, n}(\boldsymbol{S})$. Then there are $d \in \boldsymbol{D}$ and $u \in \boldsymbol{U}$ such that both $d X$ and $X u$ belong to $\mathfrak{M}_{1, n}(\boldsymbol{R})$.

Proof of Claim. Easy by induction on $n$, by using the assumption on $\boldsymbol{D}$ and $\boldsymbol{U}$, and the obvious fact that both $\boldsymbol{D}$ and $\boldsymbol{U}$ are closed under multiplication.

$\square$ Claim.

Let $n \in \mathbb{N}$ and let $U \in \mathfrak{M}_{1, n}(\boldsymbol{S})$ be a row matrix of $\boldsymbol{S}$. We prove that the solution set in $\mathfrak{M}_{n, 1}(\boldsymbol{S})$ of the following mixed system

$$
\left\{\begin{array}{r}
U X \geq 0 \\
X \geq 0
\end{array}\right.
$$

is a finitely generated $\boldsymbol{S}^{+}$-subsemimodule of $\mathfrak{M}_{n, 1}(\boldsymbol{S})$. By the Claim, there exists $d \in \boldsymbol{D}$ such that $d U \in \mathfrak{M}_{1, n}(\boldsymbol{R})$. Note that for all $X \in$ $\mathfrak{M}_{n, 1}\left(\boldsymbol{R}^{+}\right), U X \geq 0$ if and only if $d U X \geq 0$. Hence, we may assume without loss of generality that $U$ belongs to $\mathfrak{M}_{1, n}(\boldsymbol{R})$. Since $\boldsymbol{R}$ is right po-coherent, there exist $k \in \mathbb{N}$ and $P \in \mathfrak{M}_{n, k}(\boldsymbol{R})$ such that

$$
\left\{X \in \mathfrak{M}_{n, 1}\left(\boldsymbol{R}^{+}\right) \mid U X \geq 0\right\}=\left\{P Y \mid Y \in \mathfrak{M}_{k, 1}\left(\boldsymbol{R}^{+}\right)\right\} .
$$

Thus, in order to conclude the proof, it suffices to prove that the following holds:

$$
\left\{X \in \mathfrak{M}_{n, 1}\left(\boldsymbol{S}^{+}\right) \mid U X \geq 0\right\}=\left\{P Y \mid Y \in \mathfrak{M}_{k, 1}\left(\boldsymbol{S}^{+}\right)\right\} .
$$

Since $P \geq 0$ and $U P \geq 0$, the containment from the right into the left is obvious. Conversely, let $X \in \mathfrak{M}_{n, 1}\left(\boldsymbol{S}^{+}\right)$such that $U X \geq 0$. By the Claim, there exists $u \in \boldsymbol{U}$ such that $X u \in \mathfrak{M}_{n, 1}\left(\boldsymbol{R}^{+}\right)$. Since $u \geq 0$, we also have $U X u \geq 0$ and $X u \geq 0$. By definition, there exists $v \in \boldsymbol{S}^{+}$ such that $u v=1$. By (15.2), there exists $Y \in \mathfrak{M}_{k, 1}\left(\boldsymbol{R}^{+}\right)$such that $X u=P Y$. It follows that $X=X u v=P Y v$. Note that $Y v \geq 0$. This concludes the proof of (5.3).

\section{Subrings of the RAtionals}

We shall prove in this section (Theorem 6.4) that every subring of $\mathbb{Q}$ is $p o$-coherent. The hard core of this fact is the case of the ring $\mathbb{Z}$ of integers. The result follows then from a much more general principle, due, in the context of commutative semigroups, to Grillet, see [6], and, in the context of partially ordered abelian groups, to Effros, Handelman, and Shen, see [4], and also [5]. In order to formulate this result, we need a few definitions.

Definition 6.1. Let $\boldsymbol{G}$ be a partially ordered abelian group.

(i) $\boldsymbol{G}$ is directed, if $\boldsymbol{G}=\boldsymbol{G}^{+}+\left(-\boldsymbol{G}^{+}\right)$. 
(ii) $\boldsymbol{G}$ is unperforated, if $m x \geq 0$ implies that $x \geq 0$, for all $m \in \mathbb{N}$ and all $x \in \boldsymbol{G}$.

(iii) $\boldsymbol{G}$ satisfies the interpolation property, or $\boldsymbol{G}$ is an interpolation group, if for all $a_{0}, a_{1}, b_{0}, b_{1} \in \boldsymbol{G}$ such that $a_{0}, a_{1} \leq b_{0}, b_{1}$, there exists $x \in \boldsymbol{G}$ such that $a_{0}, a_{1} \leq x \leq b_{0}, b_{1}$.

(iv) $\boldsymbol{G}$ is a dimension group, if it is a directed, unperforated interpolation group.

An important particular class of dimension groups is the class of simplicial groups; a partially ordered abelian group is simplicial, if it is isomorphic to some $\mathbb{Z}^{n}$, with $n \in \mathbb{Z}^{+}$. The Grillet, Effros, Handelman, and Shen Theorem states that a partially ordered abelian group is a dimension group if and only if it is a direct limit of simplicial groups and positive homomorphisms (that is, order-preserving group homomorphisms).

Proposition 6.2. Let $m, n \in \mathbb{N}$, let $M \in \mathfrak{M}_{m, n}(\mathbb{Z})$. Then there exist $k \in \mathbb{N}$ and a matrix $S \in \mathfrak{M}_{k, m}(\mathbb{Z})$ such that for every unperforated interpolation group $\boldsymbol{G}$, the solution set in $\mathfrak{M}_{1, m}\left(\boldsymbol{G}^{+}\right)$of the system $X M \geq 0$ is exactly the set

$$
\left\{Y S \mid Y \in \mathfrak{M}_{1, k}\left(\boldsymbol{G}^{+}\right)\right\} .
$$

The case where $m=1$, sufficient to deduce the Grillet, Effros, Handelman, and Shen Theorem, is done in [5, Proposition 3.15]. The general case is easily deduced by induction, in a similar fashion as in the proof of Theorem 3.6 .

By applying Proposition 6.2 to $G=\mathbb{Z}$, we obtain the following result:

Corollary 6.3. The ring $\mathbb{Z}$ of integers, endowed with its natural ordering, is po-coherent.

By using Proposition [5.7, we thus obtain a large class of $p o$-coherent po-rings:

Theorem 6.4. Every subring of $\mathbb{Q}$ is po-coherent.

Proof. Let $\boldsymbol{R}$ be a subring of $\mathbb{Q}$. By Corollary 6.3, the ordered ring $\mathbb{Z}$ is $p o$-coherent. Since $\mathbb{Z}$ is a directed subring of $\boldsymbol{R}$, it suffices to prove that the containment of ordered rings $\mathbb{Z} \subseteq \boldsymbol{R}$ satisfies the assumption of Proposition 5.7. We define subsets $\boldsymbol{D}$ and $\boldsymbol{U}$ of $\boldsymbol{R}^{+}$as in Proposition 5.7. Then we have $\boldsymbol{D}=\mathbb{N}$, thus, since $\boldsymbol{R} \subseteq \mathbb{Q}$, (i) is obvious. Now we prove (ii). So let $x \in \boldsymbol{R}$. Write $x=p / q$, where $p \in \mathbb{Z}, q \in \mathbb{N}$ and $p$ and $q$ are coprime. By Bezout's Theorem, there are integers $u$ and $v$ 
such that $u p+v q=1$. Therefore, the equality

$$
\frac{1}{q}=\frac{u p+v q}{q}=u x+v \in \boldsymbol{R}
$$

holds, so that $q \in \boldsymbol{U}$. Moreover, $q x=p \in \mathbb{Z}$, which completes the verification of (ii).

Example 6.5. We construct a subring of the ring $\mathbb{R}$ of real numbers that is not even coherent as a ring. Let $u, v$ and $x_{n}\left(n \in \mathbb{Z}^{+}\right)$be algebraically independent elements of $\mathbb{R}$ over $\mathbb{Q}$, and let $\boldsymbol{R}$ be the subring of $\mathbb{R}$ generated by the elements $u, v$, and $u x_{n}, v x_{n}$ for all $n \in \mathbb{Z}^{+}$. Note, in particular, that no $x_{n}$ belongs to $\boldsymbol{R}$. Then it is not difficult to verify that the set $\boldsymbol{I}$ defined by

$$
\boldsymbol{I}=\{\langle x, y\rangle \in \boldsymbol{R} \times \boldsymbol{R} \mid u x=v y\}
$$

is the $\boldsymbol{R}$-submodule of $\boldsymbol{R} \times \boldsymbol{R}$ generated by all pairs $\langle v, u\rangle$ and $\left\langle v x_{n}, u x_{n}\right\rangle$, for $n \in \mathbb{Z}^{+}$, and that it is not finitely generated. Therefore, $\boldsymbol{R}$ is not coherent.

\section{Totally ORDERED DiVISION RINGS}

In the previous section, we have seen that, in particular, the ordered field of rationals is a po-coherent $p o$-ring. In this section, we shall see that every totally ordered field is po-coherent. In fact, the commutativity will not be used in the proof, so that this result will hold for totally ordered division rings.

We first state the analogue, for totally ordered division rings, of Definition 6.1.

Definition 7.1. Let $\boldsymbol{K}$ be a totally ordered division ring. A right partially ordered vector space, or, simply, a partially ordered vector space, over $\boldsymbol{K}$ is, by definition, a partially ordered right $\boldsymbol{K}$-module. A dimension vector space over $\boldsymbol{K}$ is a directed partially ordered vector space over $\boldsymbol{K}$ satisfying the interpolation property.

Note that a partially ordered vector space is automatically unperforated, so that every dimension vector space is also a dimension group. We also extend naturally to vector spaces the definition, stated in Section [6, of a simplicial group: a simplicial vector space over $\boldsymbol{K}$ is a partially ordered vector space over $\boldsymbol{K}$ which is isomorphic to some $\boldsymbol{K}^{n}$ (endowed with the natural ordering), for some $n \in \mathbb{Z}^{+}$.

The analogue for partially ordered vector spaces over $\boldsymbol{K}$ of the Grillet, Effros, Handelman, and Shen Theorem is then that for every totally ordered division $\operatorname{ring} \boldsymbol{K}$, the dimension vector spaces over $\boldsymbol{K}$ are 
exactly the direct limits of simplicial vector spaces and positive homomorphisms of $\boldsymbol{K}$-vector spaces.

Similarly, we can formulate for totally ordered division rings the following analogue of Proposition 6.2.

Proposition 7.2. Let $\boldsymbol{K}$ be a totally ordered division ring. Let $m$, $n \in \mathbb{N}$, let $M \in \mathfrak{M}_{m, n}(\boldsymbol{K})$. Then there exist $k \in \mathbb{N}$ and a matrix $S \in \mathfrak{M}_{k, m}(\boldsymbol{K})$ such that for every interpolation vector space $\boldsymbol{E}$ over $\boldsymbol{K}$, the solution set in $\mathfrak{M}_{1, m}\left(\boldsymbol{E}^{+}\right)$of the system $X M \geq 0$ is exactly the set

$$
\left\{Y S \mid Y \in \mathfrak{M}_{1, k}\left(\boldsymbol{E}^{+}\right)\right\} .
$$

Proof. The hard core of the proof consists, similarly as for Proposition 6.2, to prove that if $\boldsymbol{E}$ is an interpolation vector space over $\boldsymbol{K}$, then, for all $n \in \mathbb{N}$ and all $p_{1}, \ldots, p_{n} \in \boldsymbol{K}$, the solution set in $\left(\boldsymbol{E}^{+}\right)^{n}$ of the equation

$$
x_{1} p_{1}+\cdots+x_{n} p_{n}=0
$$

is the set of linear combinations with coefficients from $\boldsymbol{E}^{+}$of a certain finite subset of $\left(\boldsymbol{K}^{+}\right)^{n}$. Actually, the proof of this fact is much easier than for groups, because of the possibility of dividing by any nonzero element of $\boldsymbol{K}$.

Put $U=\left\{i \in\{1, \ldots, n\} \mid p_{i}>0\right\}, V=\left\{i \in\{1, \ldots, n\} \mid p_{i}<0\right\}$, and $q_{i}=-p_{i}$, for all $i \in V$. Note that $U \cap V=\varnothing$. Then (7.1) can be written as follows:

$$
\sum_{i \in U} x_{i} p_{i}=\sum_{j \in V} x_{j} q_{j}
$$

Since $\boldsymbol{E}^{+}$satisfies the refinement property (see [5, Proposition 2.2(c)]), this is equivalent to saying that there are elements $z_{i j} \in \boldsymbol{E}^{+}$, for $\langle i, j\rangle \in$ $U \times V$, such that

$$
\begin{aligned}
x_{i} p_{i}=\sum_{j \in V} z_{i j}, & \text { for all } i \in U, \\
x_{j} q_{j}=\sum_{i \in U} z_{i j}, & \text { for all } j \in V .
\end{aligned}
$$

Dividing by $p_{i}$ (resp., $q_{j}$ ) yields the following equivalent form of (7.2):

$$
\begin{array}{ll}
x_{i}=\sum_{j \in V} z_{i j} p_{i}^{-1}, & \text { for all } i \in U, \\
x_{j}=\sum_{i \in U} z_{i j} q_{j}^{-1}, & \text { for all } j \in V .
\end{array}
$$


Thus the solution set in $\left(\boldsymbol{E}^{+}\right)^{n}$ of (17.1) is the set of linear combinations with coefficients from $\boldsymbol{E}^{+}$of a subset of $\left(\boldsymbol{K}^{+}\right)^{n}$ with $n+|U| \cdot|V|-|U \cup V|$ elements.

For general $m$, the conclusion follows from an easy induction argument, similar to the one used in the proof of Theorem 3.6.

Corollary 7.3. Every totally ordered division ring is po-coherent.

\section{8. po-COHERENCE OF FINITELY PRESENTED ABELIAN}

\section{LATTICE-ORDERED GROUPS}

The main goal of this section is to provide a proof for the following result:

Theorem 8.1. Every finitely presented abelian lattice-ordered group, viewed as a partially ordered abelian group, is po-coherent.

We shall need to recall some basic results about abelian latticeordered groups. The reader may consult the survey article [7 for more information.

\subsection{Finitely generated abelian lattice-ordered groups and poly-} hedral cones. We refer to [2, 11, 12, for more information about this section. Let $n$ be a positive integer. The abelian group $\mathbb{Z}^{\mathbb{Z}^{n}}$ of all maps from $\mathbb{Z}^{n}$ to $\mathbb{Z}$, endowed with its coordinatewise ordering, is an abelian lattice-ordered group. We consider the sub-lattice-ordered group $\mathrm{FA}(n)$ of $\mathbb{Z}^{\mathbb{Z}^{n}}$ generated by the $n$ canonical projections $p_{i}: \mathbb{Z}^{n} \rightarrow \mathbb{Z}$, for $1 \leq i \leq n$. It is then well-known that $\left\langle\mathrm{FA}(n), p_{1}, \ldots, p_{n}\right\rangle$ is the free abelian lattice-ordered group on $n$ generators. Every element $f$ of $\mathrm{FA}(n)$ can be decomposed as

$$
f=\bigwedge_{1 \leq i \leq k} f_{i}-\bigwedge_{k+1 \leq i \leq k+l} f_{i}
$$

where $k, l$ are positive integers and all the $f_{i}$ are linear functionals from $\mathbb{Z}^{n}$ to $\mathbb{Z}$. In particular, $f$ can be extended to a unique positively homogeneous map from $\mathbb{Q}^{n}$ to $\mathbb{Q}$, defined by (8.1) where the $f_{i}$ are replaced by their unique extensions to linear functionals on $\mathbb{Q}^{n}$. Throughout this section, we shall identify whenever needed an element of $\mathrm{FA}(n)$ with its positively homogeneous extension to $\mathbb{Q}^{n}$.

As in [1, 7, we define a convex polyhedral cone of $\mathbb{Q}^{n}$ to be a finite intersection of closed half-spaces of $\mathbb{Q}^{n}$, where, as usual, a closed halfspace is a subset of the form $\{x \mid p(x) \geq 0\}$, where $p$ is a nonzero linear functional on $\mathbb{Q}^{n}$. Further, we define a polyhedral cone to be a finite union of convex polyhedral cones.

We recall two classical lemmas: 
Lemma 8.2. Let $n$ be a positive integer, let $f \in \mathrm{FA}(n)$, let $K$ be a polyhedral cone of $\mathbb{Q}^{n}$. Then there exist a positive integer $N$ and a decomposition $K=\bigcup_{1 \leq l \leq N} K_{l}$ of $K$ into convex polyhedral cones such that for all $l$, there exists a linear functional $f_{l}$ on $\mathbb{Z}^{n}$ such that $f \uparrow_{K_{l}}=f_{l} \uparrow_{K_{l}}$.

Proof. Without loss of generality, $K$ is a convex polyhedral cone. We decompose $f$ as in (8.1). For every permutation $\sigma$ of $\{1, \ldots, k+l\}$ that leaves both sets $\{1, \ldots, k\}$ and $\{k+1, \ldots, k+l\}$ invariant, we define $K_{\sigma}$ as the set of those $x \in K$ such that for all $i \in\{1, \ldots, k+l\}$ distinct from $k$ and from $k+l$, the inequality $f_{\sigma(i)}(x) \leq f_{\sigma(i+1)}(x)$ holds. On each $K_{\sigma}, f$ is equal to some $f_{i}-f_{j}$. The conclusion of the lemma follows, with $N=k ! l !$.

Lemma 8.3. Let $n$ be a positive integer, let $f \in \mathrm{FA}(n)$. Then the set

$$
\left\{x \in \mathbb{Q}^{n} \mid f(x) \geq 0\right\}
$$

is a polyhedral cone of $\mathbb{Q}^{n}$.

If $K$ is a polyhedral cone of $\mathbb{Q}^{n}$, a map $f: K \rightarrow \mathbb{Q}$ is piecewise linear, if there exists a decomposition $K=\bigcup_{1 \leq l \leq N} K_{l}$ of $K$ into convex polyhedral cones such that for all $l$, there exists a linear functional $f_{l}$ on $\mathbb{Q}^{n}$ which satisfies $f \uparrow_{K_{l}}=f_{l} \uparrow_{K_{l}}$. By Lemma 8.2 every element of $\mathrm{FA}(n)$ is piecewise linear on $\mathbb{Q}^{n}$. We denote by $\mathrm{PL}(K)$ the abelian lattice-ordered group of all piecewise linear maps from a polyhedral cone $K$ to $\mathbb{Q}$.

The next lemma is folklore. It can also be easily deduced from Proposition 7.2

Lemma 8.4. Let $n$ be a positive integer. Then every convex polyhedral cone $K$ of $\mathbb{Q}^{n}$ can be positively generated by a finite subset $X$, that is,

$$
K=\left\{\sum_{x \in X} \lambda_{x} x \mid \lambda_{x} \in \mathbb{Q}^{+}, \text {for all } x \in X\right\} .
$$

8.2. Finitely presented abelian lattice-ordered groups. An abelian lattice-ordered group $\boldsymbol{G}$ is finitely presented, if it is isomorphic to a quotient $\mathrm{FA}(n) / I$, where $n$ is a positive integer and $I$ is a finitely generated $\ell$-ideal of $\mathrm{FA}(n)$ (an $\ell$-ideal is a convex subgroup closed under $\wedge$ and $\vee)$. If $I$ is generated by $p_{1}, \ldots, p_{m}$, then $I$ is also generated by the single element $p=\sum_{i=1}^{m}\left|p_{i}\right|$ (where $|x|=x \vee(-x)$ for all $x$ ). Hence, $I$ takes on the following simple form

$$
I=\{f \in \mathrm{FA}(n)|\exists k \in \mathbb{N},| f \mid \leq k p\} .
$$


Put $Z(f)=\left\{x \in \mathbb{Q}^{n} \mid f(x)=0\right\}$, for all $f \in \mathrm{FA}(n)$. By Lemma 8.3. applied to $-|f|, Z(f)$ is a polyhedral cone of $\mathbb{Q}^{n}$. Put $K=Z(p)$.

We define a sub-lattice-ordered group $\boldsymbol{H}$ of $\mathrm{PL}(K)$ by

$$
\boldsymbol{H}=\left\{f \uparrow_{K} \mid f \in \mathrm{FA}(n)\right\} .
$$

There is a natural surjective homomorphism of lattice-ordered groups, $\rho: \boldsymbol{G} \rightarrow \boldsymbol{H}$, defined by the rule $\rho(f+I)=f \uparrow_{K}$, for all $f \in \mathrm{FA}(n)$. By 1. Lemma 3.3], $\rho$ is an isomorphism. We have thus outlined part of the proof of the following classical result:

Lemma 8.5. Every finitely presented abelian lattice-ordered group can be embedded into the lattice-ordered group $\mathrm{PL}(K)$ of all piecewise linear maps on a polyhedral cone $K$.

Proof of Theorem 8.1. By Lemma 8.5, in order to prove that every finitely presented abelian lattice-ordered group is po-coherent, it suffices to prove that all the groups $\operatorname{PL}(K)$ are po-coherent. Thus let $n$ be a positive integer, let $K$ be a polyhedral cone of $\mathbb{Q}^{n}$, let $f_{1}, \ldots$, $f_{m} \in \mathrm{PL}(K)$. We consider the set

$$
\mathcal{S}=\left\{\left\langle\lambda_{1}, \ldots, \lambda_{m}\right\rangle \in\left(\mathbb{Q}^{+}\right)^{m} \mid \forall x \in K, \sum_{i=1}^{m} \lambda_{i} f_{i}(x) \geq 0\right\},
$$

we must prove that $\mathcal{S} \cap \mathbb{Z}^{m}$ is a finitely generated submonoid of $\mathbb{Z}^{m}$. Since the $f_{i}$ are piecewise linear, there exist $N \in \mathbb{N}$ and a decomposition $K=\bigcup_{1 \leq l \leq N} K_{l}$ of $K$ into convex polyhedral cones such that every $f_{i}$ is linear on every $K_{l}$, that is, there exists a linear functional $f_{i, l}$ on $\mathbb{Q}^{n}$ such that $f_{i} \uparrow_{K_{l}}=f_{i, l} \uparrow_{K_{l}}$. Hence, the equality

$$
\mathcal{S}=\bigcap_{1 \leq l \leq N} \mathcal{S}_{l}
$$

holds, where we put

$$
\mathcal{S}_{l}=\left\{\left\langle\lambda_{1}, \ldots, \lambda_{m}\right\rangle \in\left(\mathbb{Q}^{+}\right)^{m} \mid \forall x \in K_{l}, \sum_{i=1}^{m} \lambda_{i} f_{i, l}(x) \geq 0\right\},
$$

for all $l \in\{1, \ldots, N\}$. However, $K_{l}$ is positively generated by a finite subset $X_{l}$ (see Lemma 8.4), so that the equality

$$
\mathcal{S}_{l}=\bigcap_{x \in X_{l}}\left\{\left\langle\lambda_{1}, \ldots, \lambda_{m}\right\rangle \in\left(\mathbb{Q}^{+}\right)^{m} \mid \sum_{i=1}^{m} \lambda_{i} f_{i, l}(x) \geq 0\right\}
$$

holds. In particular, $\mathcal{S}_{l}$ is a convex polyhedral cone, for all $l \in\{1, \ldots, N\}$, thus $\mathcal{S}$ is a convex polyhedral cone. This implies that the elements of 
$\mathcal{S} \cap \mathbb{Z}^{n}$ are the solutions in $\mathbb{Z}^{n}$ of a finite system of inequalities with coefficients from $\mathbb{Q}$, thus, after having cleared away denominators, from $\mathbb{Z}$. By Corollary 6.3, we obtain that $\mathcal{S} \cap \mathbb{Z}^{n}$ is a finitely generated monoid, which completes the proof.

Example 8.6. This example shows that the assumption that $\boldsymbol{F}$ is finitely generated convex is essential in the statement of Proposition 5.5.

For this, we consider the abelian lattice-ordered groups $\boldsymbol{G}$ and $\boldsymbol{H}$ defined as follows. First, $\boldsymbol{G}=\mathrm{FA}(2)$ is the free abelian lattice-ordered group on two generators, $x$ and $y$, and $\boldsymbol{H}=\mathbb{Z}+\alpha \mathbb{Z}$, viewed as an ordered additive subgroup of $\mathbb{R}$, where $\alpha$ is any positive irrational number. Furthermore, let $f: \boldsymbol{G} \rightarrow \boldsymbol{H}$ be the unique homomorphism of latticeordered groups sending $x$ to 1 , and $y$ to $\alpha$. By Theorem $8.1, \boldsymbol{G}$ is a po-coherent partially ordered abelian group.

Furthermore, observe that ker $f$ is an ideal (that is, a convex, directed subgroup) of $\boldsymbol{G}$, and that $\boldsymbol{H}$ is isomorphic to $\boldsymbol{G} / \operatorname{ker} f$. However, $\boldsymbol{H}$ is not a $p o$-coherent partially ordered right $\mathbb{Z}$-module. Indeed, otherwise, the following set

$$
\mathcal{S}=\left\{\langle x, y\rangle \in\left(\mathbb{Z}^{+}\right)^{2} \mid x-\alpha y \geq 0\right\}
$$

would be a finitely generated submonoid of $\left(\mathbb{Z}^{+}\right)^{2}$. Let

$$
\left\{\left\langle x_{i}, y_{i}\right\rangle \mid 1 \leq i \leq n\right\}
$$

be a finite generating subset of $\mathcal{S}$, with $n \in \mathbb{N}$ and $x_{i}>0$, for all $i$. Let $\lambda$ be the largest element of $\left\{y_{i} / x_{i} \mid 1 \leq i \leq n\right\}$. So $\alpha \lambda \leq 1$, thus, since $\alpha$ is irrational and $\lambda$ is rational nonzero, $\alpha \lambda<1$. Hence, there exists $\langle x, y\rangle$ in $\mathbb{N} \times \mathbb{N}$ such that $\alpha y / x<1$ and $\lambda<y / x$. The first inequality implies that $\langle x, y\rangle \in \mathcal{S}$, so there are nonnegative integers $k_{i}$, for $1 \leq i \leq n$, such that $x=\sum_{i} k_{i} x_{i}$ and $y=\sum_{i} k_{i} y_{i}$. In particular, $y \leq \lambda x$, so $y / x \leq \lambda$, a contradiction.

\section{Discussion}

From the results of all the previous sections, we can deduce the following:

Theorem 9.1. Let $\boldsymbol{R}$ be either a subring of $\mathbb{Q}$ or a totally ordered division ring. Let $\boldsymbol{A}$ be a partially ordered right $\boldsymbol{R}$-module. Then the following properties hold:

(i) $\boldsymbol{A}$ is finitely po-presented if and only if it is finitely related, if and only if $\boldsymbol{A}$ is a finitely presented $\boldsymbol{R}$-module and $\boldsymbol{A}^{+}$is a finitely generated $\boldsymbol{R}^{+}$-semimodule.

(ii) Suppose that $\boldsymbol{A}$ is finitely po-presented. Then every finitely generated submodule of $\boldsymbol{A}$ is finitely po-presented. 
Proof. By Theorem 6.4 and Corollary [7.3, $\boldsymbol{R}$ is a po-coherent po-ring. We conclude the proof by Theorem 4.2 for (i), and by Theorem 4.1 for (ii).

Since $\mathbb{Z}$ is a notherian ring, the particular case where $\boldsymbol{R}=\mathbb{Z}$ states that (i) a partially ordered abelian group $\boldsymbol{G}$ is finitely $p o$-presented if and only if $\boldsymbol{G}$ is a finitely generated group and $\boldsymbol{G}^{+}$is a finitely generated monoid, and (ii) every subgroup of a finitely $p o$-presented partially ordered abelian group is finitely po-presented. These results are established by completely different means in [3].

Problem 1. Let $\boldsymbol{R}$ be a po-ring, let $\boldsymbol{A}$ be a partially ordered right $\boldsymbol{R}$ module. Suppose that $\boldsymbol{A}$ is finitely po-presented. Is $\boldsymbol{A}$ finitely related?

The converse, namely the fact that finitely related implies finitely po-presented, holds as a rule, see Proposition 3.4. Furthermore, for partially ordered right modules over right $p o$-coherent rings, finitely po-presented is equivalent to finitely related, see Theorem 4.2. On the other hand, it seems unlikely that the answer to Problem 1 could be positive in general.

Problem 2. Let $\boldsymbol{R}$ be a subring of the real field $\mathbb{R}$. Suppose that $\boldsymbol{R}$ is coherent. Is $\boldsymbol{R}$ po-coherent? More generally, if $\boldsymbol{R}$ is a totally ordered ring, is it the case that coherence of $\boldsymbol{R}$ implies po-coherence of $\boldsymbol{R}$ ?

We do not know the answer even in the case where $\boldsymbol{R}$ is a notherian subring of $\mathbb{R}$, even in the case where $\boldsymbol{R}$ is an extension of $\mathbb{Z}$ by finitely many real algebraic numbers. Concretely, the problem is the following: given a coherent subring $\boldsymbol{R}$ of $\mathbb{R}$, and $n \in \mathbb{N}$ and $\alpha_{1}, \ldots, \alpha_{n} \in \boldsymbol{R}$, prove that the solution set in $\left(\boldsymbol{R}^{+}\right)^{n}$ of the equation

$$
\alpha_{1} x_{1}+\cdots+\alpha_{n} x_{n}=0
$$

is a finitely generated $\boldsymbol{R}^{+}$-subsemimodule of $\left(\boldsymbol{R}^{+}\right)^{n}$.

Problem 3. Let $\boldsymbol{R}$ be a subring of the real field, totally ordered with its natural ordering. Suppose that $\boldsymbol{R}$ is po-coherent. Does there exist an analogue of the Grillet, Effros, Handelman, and Shen Theorem that would hold for partially ordered right $\boldsymbol{R}$-modules?

Problem 4. Investigate right po-coherence of subrings of totally ordered division rings.

\section{ACKNOWLEDGMENT}

The author would like to thank the anonymous referee for having thoroughly read the paper, pointing an embarrassing number of errors and oversights, and suggesting a number of improvements. 


\section{REFERENCES}

[1] K. A. Baker, Free vector lattices, Canad. J. Math. 20 (1968), 58-66.

[2] S. J. Bernau, Free abelian lattice groups, Math. Ann. 180 (1969), 48-59.

[3] J. F. Caillot and F. Wehrung, Finitely presented, coherent and ultrasimplicial ordered abelian groups, Semigroup Forum, to appear.

[4] E. G. Effros, D. E. Handelman, and C.-L. Shen, Dimension groups and their affine representations, Amer. J. Math. 102 (1980), no. 2, 385-407.

[5] K. R. Goodearl, Partially Ordered Abelian Groups with Interpolation, Math. Surveys and Monographs 20, Amer. Math. Soc., Providence, 1986.

[6] P. A. Grillet, Directed colimits of free commutative semigroups, J. Pure Appl. Algebra 9 (1976), no. 1, 73-87.

[7] K. Keimel, Some trends in lattice-ordered groups and rings, K. A. Baker and R. Wille (eds.), Lattice Theory and its Applications. In celebration of Garrett Birkhoff's 80th birthday. Lemgo: Heldermann Verlag. Res. Expo. Math. 23, 131-161 (1995).

[8] A. I. Mal'cev, Algebraic Systems, Die Grundlagen der mathematischen Wissenschaften in Einzeldarstellungen, Band 192, Springer-Verlag, Berlin Heidelberg New York, 1973. xii+317 pp.

[9] P. Ribenboim, On ordered modules, J. Reine Angew. Math. 255 (1967), 120146.

[10] B. Stenström, Rings of Quotients, Springer-Verlag, Berlin Heidelberg New York 1975, viii+309 pp.

[11] E. C. Weinberg, Free lattice-ordered abelian groups, Math. Ann. 151 (1963), 187-199.

[12] _ Free lattice-ordered abelian groups II, Math. Ann. 159 (1965), 217222.

C.N.R.S., Département de Mathématiques, Université de Caen, 14032 Caen Cedex, France

E-mail address: wehrung@math.unicaen.fr

$U R L$ : http://www.math.unicaen.fr/ ${ }^{\sim}$ wehrung 